\title{
Desarrollo de habilidades en estudiantes de Instituciones de Educación superior para la autopresentación
}

\section{Development of skills in students of higher education institutions for self-presentation}

\author{
Marina V. Bryantseva \\ mv.bryantseva@mgou.ru \\ Moscow Region State University, Moscow, Russia. \\ https://orcid.org/0000-0002-4751-5883 \\ Olga V. Dybina \\ dybinaov@yandex.ru \\ Togliatti State University, Togliatti, Russia. \\ https://orcid.org/0000-0002-0146-3336
}

Anna A. Stryapikhina

anna-stryapikhina@rambler.ru

Nizhny Novgorod State Engineering and Economic University, Nizhny Novgorod,

Russia.

https://orcid.org/0000-0001-5837-8565

Daniil D. Melnikov

meln-alena@yandex.ru

Bashkir State University, Russia, Ufa, Russia.

Vasily L. Trostin

vasily_trostin@mail.ru

Nizhny Novgorod State Engineering and Economic University, Russia.

https://orcid.org/0000-0003-1223-5256

Recibido: $18 / 12 / 2020$

Aceptado: 14/01/2021

\section{Resumen}

El propósito del artículo fue estudiar la experiencia de desarrollar en los estudiantes de instituciones educativas profesionales la capacidad de autopresentación. La metodología presenta un estudio dirigido a diagnosticar las estrategias de autopresentación de los estudiantes. El trabajo refleja la experiencia de autopresentación de los alumnos en clases prácticas, la defensa de trabajos finales e informes en la práctica, actuaciones en concursos profesionales y congresos científicos. Los datos obtenidos en el transcurso del estudio nos permiten manifestar que la capacidad de autopresentación amplía las oportunidades laborales, contribuye a 
un aumento en la competitividad de los estudiantes de las instituciones de educación superior en el mercado laboral.

Palabras clave: tecnologías educativas, autopresentación, formación profesional, proceso educativo, competitividad.

\section{Abstract}

The purpose of the article was to study the experience of developing in students of professional educational institutions the capacity for self-presentation. The methodology presents a study aimed at diagnosing students' self-presentation strategies. The work reflects the self-presentation experience of the students in practical classes, the defense of final works and reports in practice, performances in professional competitions and scientific conferences. The data obtained in the course of the study allows us to state that the capacity for self-presentation expands job opportunities, contributes to an increase in the competitiveness of students from higher education institutions in the labor market.

Key Words: educational technologies, self-presentation, professional education, educational process, competitiveness.

\section{Introducción}

La capacidad de autopresentación en las condiciones socioeconómicas modernas es un factor significativo para aumentar la competitividad del estudiante, logrando su éxito profesional y personal. El propósito del artículo es estudiar la experiencia de desarrollar estudiantes de instituciones educativas profesionales de la capacidad de autopresentación.

La auto presentación incluye una imagen profesional, planificación de objetivos, se asocia con la conciencia del alumno de sus capacidades y deficiencias, el conocimiento de las formas de superarlas, la capacidad de expresarse, de identificar sus fortalezas y debilidades (Dobudko et al., 2019b). La capacidad de autopresentación influye en la realización de la competencia profesional. La gran importancia de la capacidad de auto organización determina la necesidad de la formación de esta capacidad en los estudiantes modernos de las instituciones de educación superior.

La formación de la capacidad de autopresentación de los estudiantes refleja el pensamiento crítico, la creatividad (resolución de problemas de manera no estándar) (Demtsura et al., 2020) la flexibilidad intelectual, emocional y conductual de los estudiantes, que se manifiestan en el aula, cuando realizando trabajos independientes, defendiendo trabajos de semestre y de licenciatura, en proceso de elaboración y presentación de proyectos, en ponencias en concursos profesionales y congresos científicos. 
La conexión entre la autopresentación y la competencia profesional se puede rastrear en la representación objetiva del estudiante de los requisitos para él del futuro campo de actividad profesional (Dobudko et al., 2019a).

La esencia de la autopresentación se revela en el proceso de demostración verbal y no verbal de la propia personalidad y presupone una autopresentación ganadora (Eliseeva et al., 2020). La tarea de la autopresentación es atraer a las personas hacia uno mismo, atraer su atención utilizando la actualización de las propias cualidades. Es decir, la capacidad de autopresentación le permite crear una impresión favorable de sí mismo como persona y como especialista competente.

La autopresentación de los estudiantes depende de la estrategia elegida. Los alumnos pueden mostrar diferentes cualidades para lograr sus propios objetivos. La tarea del docente es controlar los procesos de autopresentación de los estudiantes ya que pueden optar por estrategias negativas. Para garantizar la formación de alta calidad de la competencia profesional y la capacidad de autopresentación, es necesario un seguimiento oportuno de la situación.

\section{Marco teórico}

Hasta la fecha, la formación de la capacidad de autopresentación es revelada por una amplia gama de autores. Entre ellos se encuentran I. Jones, T. Pittman, R. Cialdini, O.A. Pikulev y otros.

La autopresentación se considera a la par con conceptos tales como gestión de impresiones, autopresentación, imagen, reputación. Al mismo tiempo, la imagen y la reputación se incluyen en los objetivos de la autopresentación (Vaganova et al., 2020c).

La capacidad de autopresentación permite alcanzar ciertos modelos, ideales (personales, profesionales y otros), tras lo cual el alumno se demuestra frente a una determinada audiencia, utilizando diversos medios de comunicación (Arbeláez-Campillo \& Rojas-Bahamon, 2020).

Hay tres componentes importantes en la formación de la capacidad de autopresentación (Gruzdeva \& Tukenova, 2019). Entre ellos se encuentra el sujeto que se presenta, el sujeto al que se presentan y el contenido que se presenta. El éxito de la autopresentación depende en gran medida de la formación de competencias y la naturaleza del desempeño por parte del sujeto del rol que desempeña (Ivanov et al., 2020). La autopresentación se evalúa en este caso de acuerdo con los siguientes criterios: el grado de conformidad del rol con el modelo (Kisel et al., 2020), la saturación con las características individuales, el rango de medios utilizados para este rol, la corrección de la interpretación de roles (Pichugina \& Bondarchuk, 2019).

Dado que los requisitos de competitividad, que dependen de la capacidad de autopresentación, están sujetos a cambios activos, se requiere un monitoreo constante 
del proceso de formación de la capacidad de autopresentación (Vaganova et al., 2020a).

La autopresentación es la presentación por parte de los sujetos de educación de sí mismos a los modos de acción disponibles social y culturalmente aceptados y. En el proceso de autopresentación, el alumno utiliza ciertas estrategias que desarrolla para formar las ideas de los demás sobre sí mismo (Pinkovetskaia et al., 2020). La estrategia de picking se refleja como el comportamiento de un individuo, caracterizado por la determinación para lograr los resultados deseados y la producción de una impresión adecuada, utilizando ciertas tácticas de autopresentación (Vaganova et al., 2020b). Una táctica es un acto conductual a corto plazo que contiene técnicas verbales y no verbales destinadas a crear una impresión para lograr objetivos a corto plazo (Pikuleva, 2013).

Según SA Karase, la capacidad de los estudiantes para la actividad de autopresentación es una cualidad integradora de los estudiantes, que se forma en el proceso de dominar todos los elementos estructurales de esta actividad (componentes cognitivos, de valor motivacional y de actividad-comportamiento) (Karaseva, 2013).

La autopresentación y la competitividad están íntimamente relacionadas entre sí y con el tiempo cambian los requisitos para su formación, por lo que, la mejora continua y la actualización de las formas de organizar este proceso, se requiere la elección de tecnologías adecuadas.

\section{Metodología}

El estudio tiene como objetivo estudiar el proceso de formación de la capacidad de autopresentación de los estudiantes como factor que aumenta la competitividad de los estudiantes en el mercado laboral. El estudio requirió una amplia muestra de estudiantes de tres universidades del país. Al elegir diferentes instituciones de educación superior, aseguramos una mayor confiabilidad de los resultados.

Para estudiar la capacidad de autopresentación de los estudiantes en su trabajo, la "Escala de tácticas de autopresentación" de O.A. Pikuleva, que incluye una lista de 64 declaraciones. Los estudiantes deben expresar el grado de acuerdo con las declaraciones dadas en una escala del 1 al 9 (más consistente con el comportamiento de un estudiante en particular). Donde 1 punto se manifiesta muy raramente en el comportamiento, 9 puntos - muy a menudo. Las declaraciones se dividen en varios grupos de tácticas de autopresentación (tipo defensivo y asertivo (independencia de la influencia de evaluaciones externas). Cada táctica incluye números específicos y

número de declaraciones. Se distinguen las siguientes estrategias: evasión, comportamiento atractivo, autoexaltación, autodespreciación, influencia contundente. 
En la tabla 1 se muestra un fragmento del cuestionario "Escala de tácticas de autopresentación" (Pikuleva, 2013).

Tabla 1. Fragmento de la encuesta "Escala de autopresentación tácticas" OA Pikuleva

\begin{tabular}{ll}
\hline No. & \multicolumn{1}{c}{ Declaración } \\
\hline $\mathbf{1}$ & Cuando es necesario, para lograr mis intereses, me utilizo la fuerza contra las personas \\
\hline $\mathbf{2}$ & Para reducir las negativas reacciones de la gente, yo empiezo a justificar mis acciones. \\
\hline $\mathbf{3}$ & Le hablo a la gente de mis logros en asuntos difíciles. \\
\hline $\mathbf{4}$ & Yo puedo utilizar mis puntos débiles por los que buscan el afecto de los demás. \\
\hline $\mathbf{6}$ & A menudo les pido ayuda a los demás \\
\hline $\mathbf{7}$ & $\begin{array}{l}\text { Para ser aceptado por el equipo, estoy de acuerdo con cualquier opinión compartida } \\
\text { por el equipo. }\end{array}$ \\
\hline $\mathbf{8}$ & Yo hago excusas de antemano si me siento que voy a fallar. \\
\hline $\mathbf{9}$ & Me esfuerzo por ser un ejemplo de cómo debe comportarse la gente. \\
\hline $\mathbf{1 0}$ & Me esfuerzo para maquillaje reparar cada herir personas sufren a causa de mí \\
\hline $\mathbf{1 1}$ & Yo siempre menciono el valor de las cosas que hablo acerca \\
\hline $\mathbf{1 2}$ & Me expresar una abierta deseo de tener las personas imitan a mí por ser un positivo \\
\hline $\mathbf{1 3}$ & ejemplo \\
\hline $\mathbf{1 4}$ & Yo puedo extoy indeciso y quiero que otros tomen la responsabilidad de una común causa \\
\hline $\mathbf{1 5}$ & Yo ayudo a otros porque yo saben que van a ayudar a mí \\
\hline $\mathbf{1 6}$ & Cuando me siento ansioso que interfiere con mi rendimiento \\
\hline $\mathbf{1 7}$ & Para hacerse querer a la gente a mí, me felicito ellas \\
\hline $\mathbf{1 8}$ & Me obstaculizar mi éxito \\
\hline $\mathbf{1 9}$ & Yo intimido a otros aplicando presión \\
\hline $\mathbf{2 0}$ & Me arrepiento de que cuando hago algo mal \\
\hline
\end{tabular}

Esta tabla muestra las declaraciones que son responsables de cada grupo de tácticas.

Para evaluar la actividad y comportamiento de los estudiantes en el proceso de auto presentación se utiliza la observación pedagógica y la evaluación experta de la actividad e independencia de los estudiantes (Klimenko et al., 2018).

\section{Resultados y discusión}

El uso de tecnologías modernas en la formación de la capacidad de autopresentación asegura el rápido logro de resultados, por lo que las tecnologías educativas más relevantes se utilizan en la formación de estudiantes de instituciones de educación superior.

Las tecnologías de proyectos, discusiones, juegos, información y casos permiten a los estudiantes activar su independencia e incluirlos en actividades cognitivas independientes. En el proceso de preparación para la defensa de las obras, los estudiantes dominan la técnica de presentación de información, demuestran sus 
competencias profesionales, su capacidad para resolver problemas profesionales y la capacidad para presentar los resultados obtenidos.

Las tareas para los estudiantes se proporcionan tanto para la perspectiva a largo plazo como para el logro operativo de las metas. La mayoría de los estudiantes activos pueden expresarse durante la presentación de los proyectos, por participar en diversas conferencias y competiciones. Preparación para el proyecto se lleva a cabo durante varias semanas, durante las cuales el estudiante de forma independiente selecciona la información, análisis de materiales, selecciona la más pertinentes, interactúa con el profesor y compañeros de estudios, y prepara los materiales de presentación. La actuación final del alumno demuestra su capacidad para presentar de forma sucinta y sencilla los resultados y logros, permite formarse una idea de él como futuro especialista y de su personalidad.

La Figura 1 muestra el proceso de protección del trabajo del proyecto en la etapa final del estudio de la disciplina. Utilizando tecnologías multimedia, los alumnos presentan el material que han estado preparando durante varias semanas. La actuación de cada alumno se realiza dentro de las reglas estrictamente establecidas.

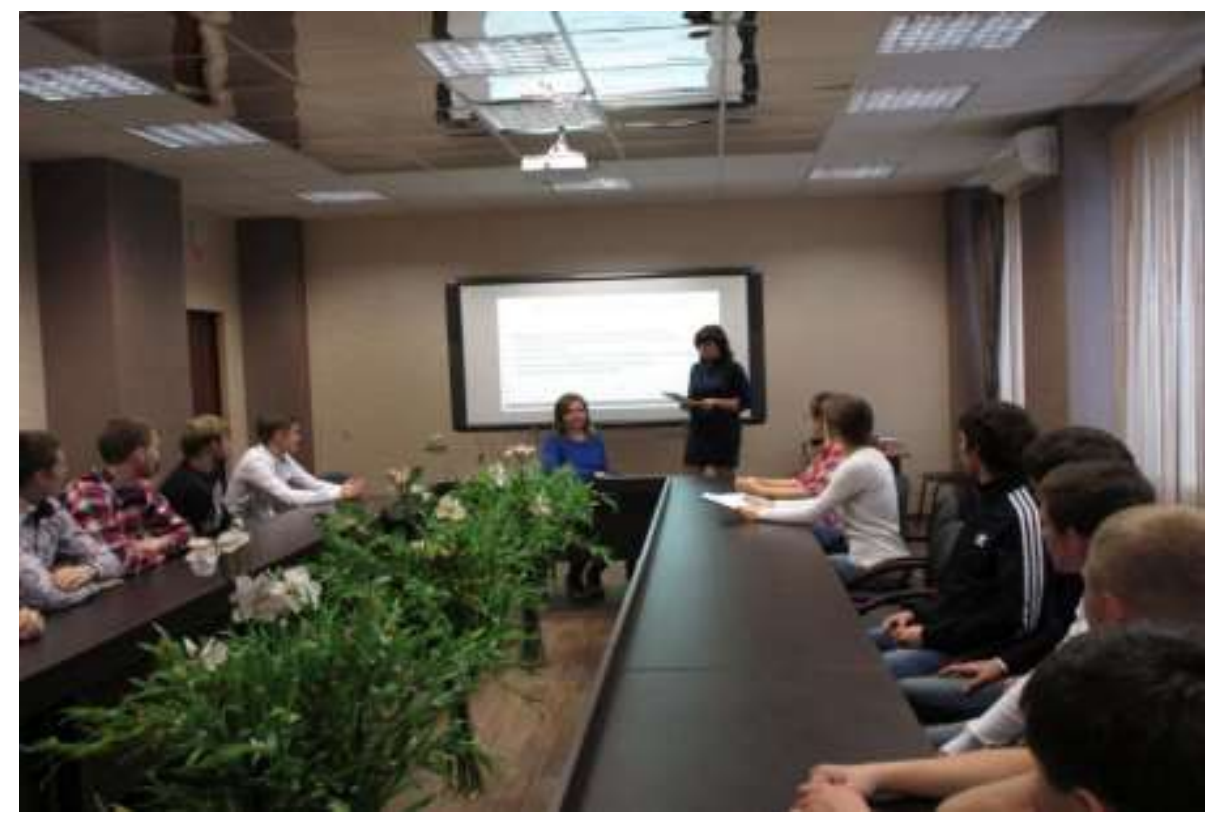

Fig. 1. Presentación de proyectos en educación superior (como parte de nuestra investigación)

Durante las discusiones en el aula, muchos estudiantes demuestran abiertamente elementos de intimidación para lograr sus propios objetivos, interrumpiendo a sus oponentes. Para evitar tales situaciones, el maestro establece reglas según las cuales los estudiantes reciben puntos de penalización por su infracción. Durante la discusión 
de cuestiones generales, todos tienen derecho a expresarse. Alguien usa técnicas de justificación, evitando la responsabilidad. Otros estudiantes se caracterizan por la conformidad. La activación de la actividad cognitiva de los alumnos y la inclusión en la actividad activa de cada alumno se realiza a través de las tecnologías de la información y la comunicación. Los estudiantes, interactuando, seleccionan la información necesaria, resuelven conjuntamente problemas emergentes, desarrollan el pensamiento crítico, la capacidad de ref lección, habilidades de comunicación que son de gran importancia para la formación de la capacidad de autopresentación.

siguiendo una determinada estrategia (estrategia de evasión, comportamiento atractivo, autoengrandecimiento, auto humillación, influencia de poder). Identificación oportuna de los tipos negativos de las estrategias se permitirá a ajustar la enseñanza de procesos y la capacidad de auto presentes estudiantes.

Para probar la capacidad de autopresentación, la "Escala de tácticas de autopresentación" fue utilizada por O. A. Pikuleva, que reveló los tipos de tácticas de autopresentación disponibles para los estudiantes: evasión, comportamiento atractivo, autoengrandecimiento, auto humillación, influencia de poder. A los estudiantes se les entregó un cuestionario que contenía varias decenas de afirmaciones, con lo cual se les pidió que expresaran un grado de acuerdo en una escala del 1 al 9 para interpretar los resultados, a cada afirmación específica se le asigna un cierto número de puntos. La figura muestra los resultados del procesamiento estadístico de los datos recibidos de estudiantes de instituciones de educación superior.

La Figura 2 muestra los resultados del procesamiento estadístico de datos sobre las estrategias de auto presentación de los estudiantes. 


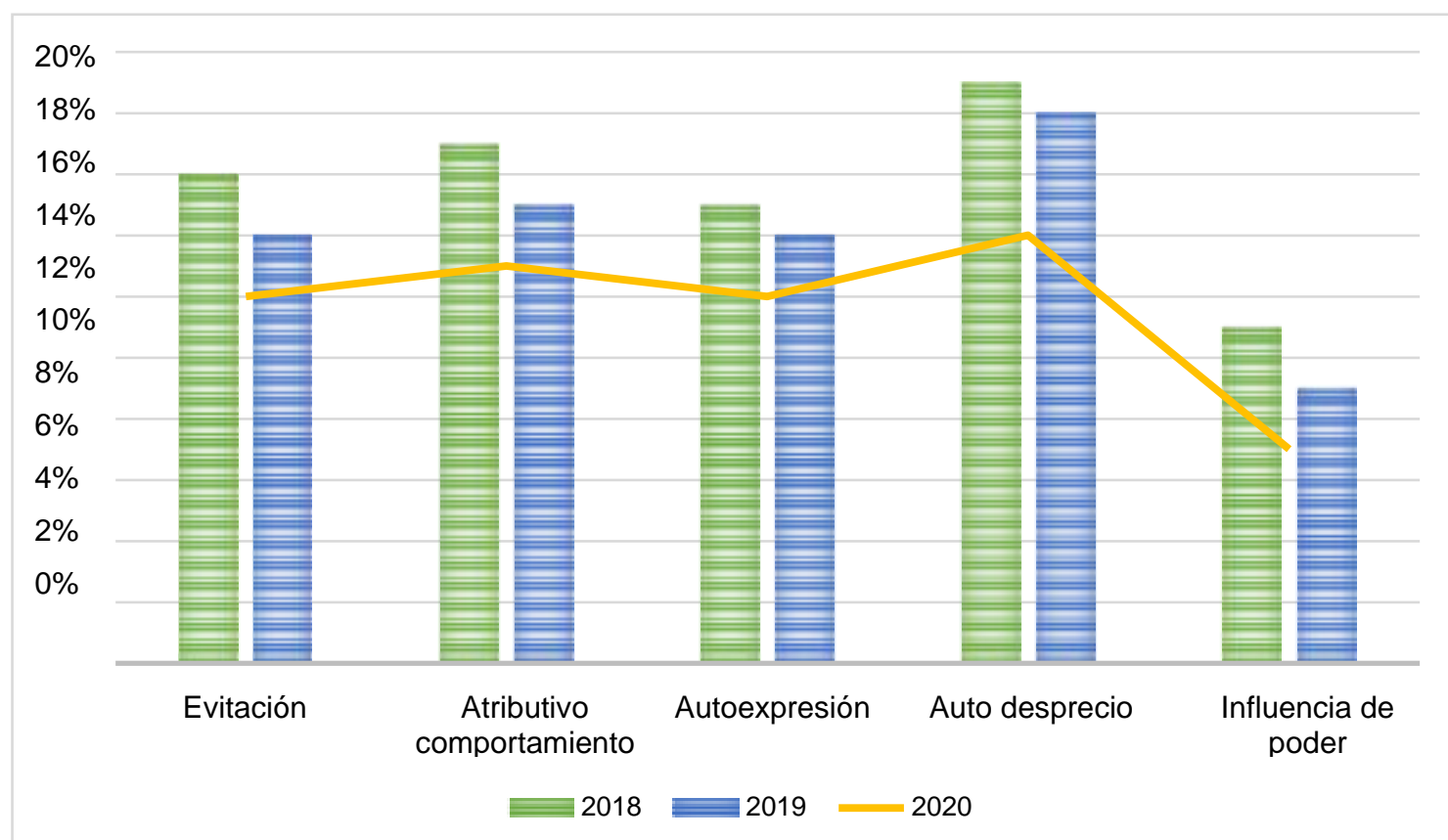

Fig. 2. Resultados del procesamiento de datos estadísticos para identificar la estrategia de auto presentación de los estudiantes (como parte de nuestra investigación)

La estrategia de evasión incluye una táctica de negación de responsabilidad (es decir, un tipo de táctica defensiva). Comportamiento atractivo que evoca una impresión favorable del tema de la auto- presentación incluye asertivas tácticas. El estudiante, a través de la adulación y que proporcionan servicios, se esfuerza para mirar mejor en frente de otras materias. La estrategia de auto- engrandecimiento se caracteriza por una demostración de alta autoestima. El alumno declara abiertamente sus méritos y logros, y habla de sus resultados de manera más positiva que ellos. El signo principal de una estrategia de autodesprecio es una demostración de debilidad. Para recibir ayuda, el alumno muestra desamparo y dependencia. Los estudiantes que eligen una estrategia de influencia contundente demuestran su poder y estatus a través del acoso (un tipo de táctica asertiva).

Podemos notar que, durante el estudio, del número total de encuestados, el número de estudiantes que utilizan estrategias de autopresentación negativas disminuye gradualmente.

\section{Conclusiones}

Las tecnologías implementadas en la formación de estudiantes de instituciones de educación superior contribuyen a la formación de la capacidad de autopresentación de los estudiantes. 
El método utilizado para identificar las estrategias de autopresentación de los estudiantes permitió establecer una reducción en la elección de estrategias negativas. Las tecnologías educativas utilizadas en la formación de los estudiantes contribuyen a la corrección oportuna del proceso educativo de acuerdo con las necesidades de los estudiantes.

La dinámica estudiados más de tres años muestra una disminución en el número de estudiantes que buscan para su uso evasión, atractivo comportamiento, autoengrandecimiento, autodesprecio, y la fuerza de influencia.

La capacidad de autopresentación amplía las oportunidades de los estudiantes para desarrollar la competencia profesional y contribuye a incrementar la competitividad en el mercado laboral, ya que un estudiante que está listo para la autopresentación es capaz de evaluar objetivamente sus ventajas y desventajas. Por lo tanto, dicho estudiante está listo para la superación personal profesional en un entorno en constante cambio.

\section{Referencias}

Dobudko, T.V., Korostelev, A.A., Gorbatov, S.V., Kurochkin, A.V., \& Akhmetov, L.G. (2019a). The organization of the university educational process in terms of digitalization of education. Humanities and Social Sciences Reviews, 7(4), 1148-1154.

Eliseeva, D.Yu., Fedosov, A.Yu., Agaltsova, D.V., Mnatsakanyan, O.L., \& Kuchmezov, KH.KH. (2020). The evolution of artificial intelligence and the possibility of its application in cyber games. Amazonia Investiga, 9(28), 123-129. https://amazoniainvestiga.info/index.php/amazonia/article/view/1043

Gruzdeva, M.L., \& Tukenova, N. I. (2019). Analysis of the current state of research and development in the construction of information and educational environments of higher educational institutions. Vestnik of Minin University, 7(2),1. (in Russ.).

Ivanov, M.S., Parnikova, T.A., Gulyaev, V.P., \& Petrov, N.V. (2020). The activity approach implementation in the formation of students' general technical $\begin{array}{llll}\text { competencies. } \quad \text { Amazonia } & \text { Investiga, 265-210. }\end{array}$ https://amazoniainvestiga.info/index.php/amazonia/article/view/1138

Karaseva, S. A. (2013). Model of the process of forming students' readiness for selfpresentation activities. Bulletin of the Adygeya State University. Series 3: Pedagogy and Psychology, 3(123), 48-53.

Kisel, O.V., Dubskikh, A. I., \& Butova, A. V. (2020). Advantages of using a studentcentered approach in higher education. Baltic Humanitarian Journal, Vol. 9, № 4 (33), 97-100. (in Russ.).

Klimenko, A.A., Khazova, S. A., \& Karaseva, S. A. (2018). Self-presentation competence: content and basics of formation. Tutorial, 162.

Pichugina, G.A., \& Bondarchuk, A.I. (2019). Structure of the training case in the organization of the educational process. Humanitarian Balkan Research, 2(4), 5-7. 
Pikuleva, O. A. (2013). Psychological ambiguity of the concept of "self-presentation of the individual" and modern scientific approaches to understanding its content. Social Psychology and Society, 2, 21-34.

Pinkovetskaia, I.S., Arbeláez-Campillo, D.F., Rojas-Bahamón, M. J., \& Veas Iniesta, D.S. (2020). Motivation of new entrepreneurs in modern economies. Amazonia Investiga, $9(29)$, 368-373. https://amazoniainvestiga.info/index.php/amazonia/article/view/1403

Vaganova, O. I, Kutepov M. M., Chelnokova E. A., Harabadjah, M. N., \& Merzlyakova, N. S. (2020c). Technology for successful training in vocational education. Revista de la Universidad del Zulia, 11(31), 473-484.

Vaganova, O. I., Petrozitskaya, I. A., Snatovich, A. B., Odarich, I. N., \& Kirillova, I. K. (2020a). Heuristic technologies of training in professional education. Amazonia Investiga, 9 , (27), 509-517. https://amazoniainvestiga.info/index.php/amazonia/article/view/1265

Vaganova, O. I., Bakharev, N. P., Bulaeva, M. N., Lapshova A. V., \& Kutepov, M. M. (2020b). Methods and means of realization of interaction technologies in the university. Amazonia Investiga, $9(26)$, 383. https://amazoniainvestiga.info/index.php/amazonia/article/view/1168 